\title{
Backscattering factor for some personal dosimeters and impact on $\mathrm{Hp}(\mathbf{1 0})$
}

\author{
V.P. SINGH ${ }^{1, *}$, N.M. BADIGER ${ }^{1}$, R.R. BIHARI ${ }^{2}$
}

(Manuscript received 29 May 2012, accepted 19 November 2012)

\begin{abstract}
In the present paper, we estimated backscattering factors for the TLD and active personal dosimeters using $662 \mathrm{keV}$ gamma-ray photons on a PMMA surface, which are, for the TLD, 1.12; Dosicard, 1.116; DRD, 0.99; and Saphydose, 1.073, and on a water slab phantom surface; for the TLD, 1.115; Dosicard, 1.112; DRD, 1; and Saphydose, 1.059. The backscattering factor contribution for the Saphydose is nearly half that of the TLD and Dosicard, whereas it is zero for the DRD. The DRD underestimates $H p(10)$ by $10.3-11.6 \%$, whereas for the Saphydose it is 3.4-5\% compared with the TLD or Dosicard. The response of the DRD to $H p(10)$ is nearly independent of the phantom, whereas the TLD, Dosicard and Saphydose require phantoms for calibration for personal monitoring. It is observed that the PMMA phantom contributes slightly higher backscattering compared with the water slab phantom. The backscattering factor of the selected dosimeters is comparable with ICRU tissue already reported.
\end{abstract}

Keywords: air kerma / backscattering / operational quantity / ambiguity / photon and $\mathrm{Z}_{\text {eff }}$

\section{Introduction}

Personnel gamma exposure monitoring in nuclear facilities is carried out by two types of personal dosimeters; passive and active. The $\mathrm{CaSO}_{4}$ : Dy Thermoluminescence Dosimeter (TLD) is used as a passive dosimeter (Vohra et al., 1980) for personnel monitoring and record-keeping in India. The personal dosimeter is worn on the chest level as representative of whole-body exposure measurement. Various studies have been carried out for the body scattered photon contribution to the personal dosimeter by using conversion factors. Two dosimeter algorithms have also been developed for estimation of the realistic effective dose (Lakshmanan et al., 1991; Kim et al., 1999). Backscattered photons are due to single and multiple scattering events (Mizukami et al., 1967) by body constituent elements. The quantitative estimation of the dose delivered by direct and backscattered photon

\footnotetext{
* Permanent address: Health Physics Section, Kaiga Atomic Power Station-3\&4, NPCIL, Karwar, 581400, Karnatak, India, e-mail: kudphyvps@ rediffmail.com

Department of Physics, Karnatak University, Dharwad, 580003, India

2 Radiological Physics \& Advisory Division, BARC, Mumbai, 400094, India.
} 
contributions is required for performance evaluation of the operational quantity measured by the dosimeter. The criterion for personnel dosimetry performance is described in ANSI-1983/1993 (ANSI, 1993).

The backscattering factor (BSF) of the phantoms has been derived at different photon energies (Traub et al., 1997). The dose measurement by dosimeters in-air, and on the phantom surface, depends on the gamma photon energy, density of the medium and $Z_{\text {eff }}$ for interaction. The dosimeters measure air kerma $\left(K_{\mathrm{a}}\right)$ when placed in air but provide the equivalent dose when placed on the phantom surface. The $K_{\mathrm{a}}$ measured under standard conditions is less than the dose equivalent. The reason is that when the dosimeter is placed on the phantom surface, simulating practical wearing on the chest, backscattered photons contribute to the dose estimation. The BSF for the dosimeters may not be similar because of their mechanism for equivalent dose measurement, material contents, the active volume of the dosimeter, and the effective area for backscattered photons, backscattered photon energy, and the distance between the dosimeter and the backscattering surface. According to HPS N13.11-1993, the ${ }^{137}$ Cs conversion factor from air kerma to the dose equivalent is $1.21 \mathrm{rem}^{\mathrm{rad}} \mathrm{d}^{-1}$ in air, and air kerma to exposure equivalency is 0.878 rad.air.kerma.exposure ${ }^{-1}$. Various studies for the BSF have been reported earlier (Chan and Doi, 1960; Kramer et al., 2001; Bartlett et al., 1990; Scott and Thomas, 2008; Gualdrini et al., 1999; Grosswendt, 1990; Selbach et al., 1985; Gualdrini, 1993). However, no study is found in the literature for active dosimeters.

The BSF depends on the backscattering material, photon energy and detector used for exposure measurement. In personal monitoring programs, various types of dosimeters of different effective atomic numbers are used, which modify the photon interaction process. Therefore, the BSF of a phantom is expected to be changed for a particular dosimeter. The BSF of a phantom for a dosimeter is an important parameter for accurate dose estimation in terms of operational quantity, $H p(10)$. Since the number of multiple backscattered events increases with decrease in photon energy and is saturated with an increase in target thickness (Sabharwal et al., 2009a, 2009b; Akar et al., 2012), backscattered photons from a phantom are expected to be optimum at $662 \mathrm{keV}$ calibration photon energy.

Free-in-air kerma measurement by dosimeters is used for quality assurance and quality control of the dosimeters. The International Commission of Radiation Units (ICRU, 1985) has recommended the dose equivalent for personal monitoring specified at a depth in the ICRU sphere (ICRU, 1980) and the personal dosimeter should be calibrated under simplified conditions of an appropriate phantom, such as the ICRU sphere. Individual dose quantities are defined at $10 \mathrm{~mm}$ and $0.07 \mathrm{~mm}$ depth inside the human body, which is not possible for all types of dosimeters. We used $30 \times 30 \times 15 \mathrm{~cm}^{3}$ water slab and PMMA phantoms for calibration of the 
dosimeters, as recommended by the American National Standard Institute (ANSI, 1993), which show closer backscattered radiation (Bartlett et al., 1990).

The BSF for personal dosimeters is essential for nuclear facilities to estimate uncertainty in dose measurement for a large range of dose receivers and collective doses. The BSF for active personal dosimeters, TLDs, and its impact on $H p(10)$ were investigated in the present study. We discuss the impact of the BSF on $H p(10)$ measurement for the most abundant photon energy in nuclear reactor technology.

\section{Material and methods}

A passive dosimeter, $\mathrm{CaSO}_{4}$ : Dy Teflon-based three-disc TLD badge, and active dosimeters; the DRD, Dosicard and Saphydose, were used for estimation of the BSF on PMMA and water phantom surfaces. $\mathrm{CaSO}_{4}$ : Dy TL dosimeter and BARC developed reader were used for dose evaluation. The Dosicard is an energycompensated silicon-based electronic dosimeter, with a large non-volatile memory and programmable alarm levels for the dose and dose rate, and lightweight, which measures gamma/X-rays according to ICRU-39 with certification in compliance with IEC-1283. The DRD is a rugged, fountain-pen-sized, conductive-fiber electroscopic dosimeter with an ion chamber for detection of photons of $16 \mathrm{keV}$ to $6 \mathrm{MeV}$. The DRD has an accuracy of $\pm 10 \%$ of true exposure and is dose rateindependent. The Saphydose dosimeter is an individual, pocket-sized dosimeter displaying the individual dose equivalent $H p(10)$ for gamma/X-ray photons in the energy range of $50 \mathrm{keV}$ to $6 \mathrm{MeV}$ and for $\mathrm{X}$-rays from $2 \mathrm{MeV}$ in compliance with IEC 61283. Standard $30 \times 30 \times 15 \mathrm{~cm}^{3}$ water and PMMA phantoms were chosen as recommended by the American National Standard Institute (ANSI, 1993), showing closer backscattered radiation (Bartlett et al., 1990) with ${ }^{137} \mathrm{Cs}$ source.

Firstly, air kerma is measured by the passive and active dosimeters separated at a distance of 1 meter away from the radioactive source. Secondary, the dose is measured with the water and PMMA phantoms behind the dosimeter, keeping a similar distance from the dosimeter. Water slab and PMMA phantoms of standard size and the ${ }^{137} \mathrm{Cs}$ source were made collinear. The phantoms were removed without disturbing the frame and the dosimeters were placed around the middle of the surface with the help of the frame and thin adhesive tape for free-in-air kerma measurement.

\section{Theoretical background}

Dose conversion coefficient (Will, 1991) is given as:

$$
H p(10) / K_{\text {air }}=B S F \times T^{\mathrm{s}}(d)\left(\frac{\mu_{\mathrm{en}}}{\rho}\right)_{\text {air, } d}^{\text {PMMA }}
$$




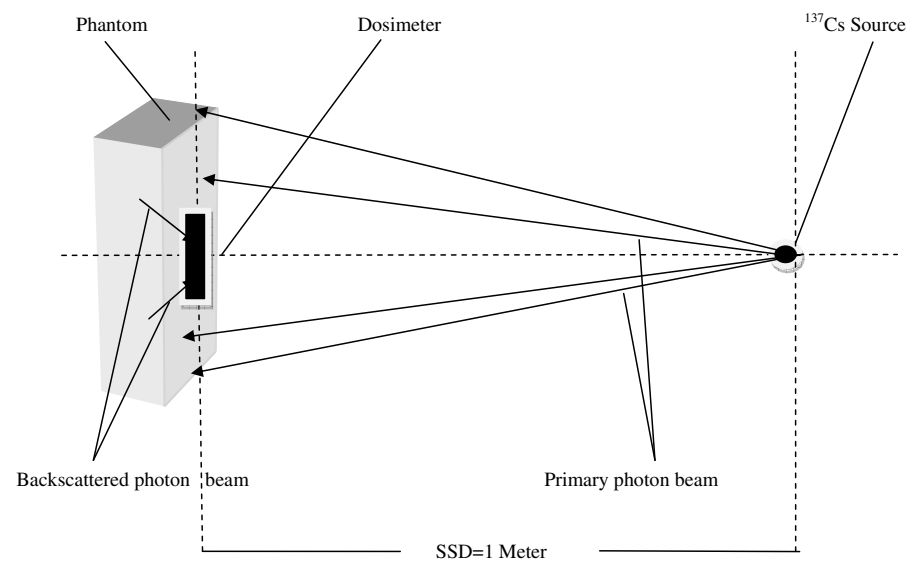

Figure 1 - Experimental set-up for backscattering factor measurement.

where

$$
B S F=\frac{\mathrm{K}_{\mathrm{air}}^{\mathrm{s}}(0)}{\mathrm{K}_{\mathrm{air}}}
$$

$T^{\mathrm{s}}(d)=\frac{\mathrm{K}_{\mathrm{air}}^{\mathrm{s}}(d)}{\mathrm{K}_{\mathrm{air}}^{\mathrm{s}}(0)}$ is the dose factor at depth $d$, and $\left(\frac{\mu_{\mathrm{en}}}{\rho}\right)_{\text {air, } d}^{\text {PMMA }}$ is the ratio of the mass energy absorption coefficient of PMMA tissue to that of the air, averaged over the photon spectrum at phantom depth, $d$. Kerma and the mass energy absorption coefficient are two parameters which are used for estimation of the BSF of dosimeters on phantoms. $\mathrm{K}_{\text {air }}^{\mathrm{s}}(0)$ is the air kerma measured at the phantom surface, and $\mathrm{K}_{\text {air }}$ is the air kerma at the same point in space in the absence of the phantom. The BSF will have an uncertainty resulting from the spectral distribution of the photon fluence and backscattered photon energy spectra with and without the phantom, on the ratio of the mass energy absorption coefficients. The magnitude of this uncertainty depends on how much photon energy spectra differ from the original energy of the photons. Equation (2) shows that the BSF of a dosimeter on a phantom can be measured if the air kerma of a dosimeter is measured for particular gamma photon energy.

\section{Experimental set-up}

The experimental set-up is shown in Figure 1, which contains water slab and PMMA phantoms arranged on opposite sides of a calibrated ${ }^{137} \mathrm{Cs}$ source such that 
TABLE I

Response of dosimeters at $662 \mathrm{keV}$ photon energy.

\begin{tabular}{lccc}
\hline Dosimeter & free-in-air & Response of personal dosimeter & water \\
\hline TLD & $1.003 \pm 0.036$ & $1.124 \pm 0.058$ & $1.119 \pm 0.019$ \\
Dosicard & $1.001 \pm 0.031$ & $1.117 \pm 0.034$ & $1.114 \pm 0.031$ \\
DRD & $1.058 \pm 0.028$ & $1.044 \pm 0.042$ & $1.060 \pm 0.035$ \\
Saphydose & $1.050 \pm 0.008$ & $1.113 \pm 0.005$ & $1.105 \pm 0.004$ \\
\hline
\end{tabular}

the midpoint of the phantoms and the source are collinear with each other. The phantom's source-to-surface distance was maintained at 1 meter. The height of the source is chosen as 1 meter from the ground's surface to make the dosimeters, phantoms and source collinear. The TLD, Dosicard, DRD and Saphydose were placed at the phantom surface and fixed with the help of adhesive tape. However, the freein-air exposure is taken by replacing the phantoms and hanging the dosimeters at a source-to-surface distance (SSD) of 1 meter. The complete arrangement was placed in the center of the room to minimize the interference of multiple scattered photons and backscattered photons from the walls of the calibration facility.

\section{Results and discussion}

\subsection{Backscattering factor}

Table I shows the normalized response of delivered air kerma for the TLD, Dosicard, DRD and Saphydose. The responses of the DRD and Saphydose were observed to be of the same order, and similarly, the TLD and Dosicard show insignificant variation. It is observed that the responses of the TLD, Dosicard and Saphydose increased by $10-12 \%$ for the water slab and PMMA phantoms, whereas the DRD had an invariable response. The reason for the insignificant response of the DRD may be the isotropic exposure of the DRD. The nil response of the DRD against backscattered photons shows that the calibration of the DRD in free-in-air exposure is representative of the field operational quantity; however, the TLD, Dosicard and Saphydose require calibration on the phantom surface $H p(10)$ simulation.

The photon interaction process is governed by photon energy and the $Z_{\text {eff }}$ of the detector material. Therefore, the $Z_{\text {eff }}$ of the dosimeter plays another vital role in comparative analysis. It is expected that dosimeters with identical $Z_{\text {eff }}$ should have a similar response. Since the $Z_{\text {eff }}$ of the TLD, Dosicard and Saphydose are of equal range $(\sim 14)$, therefore a similar response should be observed for $662 \mathrm{keV}$ photon 
TABLE II.

Backscattering factor at $662 \mathrm{keV}$ photon energy.

\begin{tabular}{lcccc}
\hline \multirow{2}{*}{ Dosimeter } & \multicolumn{2}{c}{ Back Scattering factor } & \multicolumn{2}{c}{ Relative to ICRU tissue } \\
& PMMA & water & PMMA & water \\
\hline TLD & 1.120 & 1.115 & 1.018 & 1.014 \\
Dosicard & 1.116 & 1.112 & 1.015 & 1.011 \\
DRD & 0.990 & 1.000 & 0.900 & 0.909 \\
Saphydose & 1.073 & 1.059 & 0.975 & 0.963 \\
\hline
\end{tabular}

energy. However, the response of the Saphydose was not similar to the TLD and Dosicard under the standard conditions of the experiment. It was also found that the response to delivered air kerma is within 5-6\% for all the personal dosimeters.

In Table II, the BSF for the DRD is approximately constant ( unity) for both water slab and PMMA phantoms. The experimental data shows that the DRD does not account for backscattered photons from either of the phantoms. Except for the $\mathrm{DRD}$, all the other dosimeters responded to the backscattered photon radiation; therefore, $H p(10)$ measurements would depend on the choice of the phantom. The TLD and Dosicard show nearly full response to backscattered radiation, while the Saphydose has a partial response to backscattered photons of $662 \mathrm{keV}$. The BSF for the TLD is the highest, 1.115; while that of the Saphydose is the lowest, 1.059. The $\mathrm{BSF}$ of the LiF for the PMMA to the ICRU sphere for $662 \mathrm{keV}$ photon energy is 1.06 , measured ratio and 1.03, calculated ratio. BSF calculations have been performed for a photon energy range of 10 to $2000 \mathrm{keV}$ using MCNP 4A code for the ICRU, ISO, PMMA, tissue-equivalent plastic, a combination of tissue-equivalent plastic (RS-1) with the PMMA (RS-1 \& PMMA) and Teflon (Traub et al., 1997).

Our results for the BSF for personal dosimeters are in good agreement with the MCNP 4A calculation of the BSF for $600 \mathrm{keV}, 1.103$ for ICRU; 1.095 for ISO $\left(f_{\text {ICRU }}=0.992\right) ; 1.103$ for PMMA $\left(f_{\text {ICRU }}=1\right) ; 1.093$ for RS-1 $\left(f_{\text {ICRU }}=0.991\right)$; 1.098 for RS-1\&PMMA $\left(f_{\text {ICRU }}=0.995\right)$ and 1.107 for Teflon $\left(f_{\text {ICRU }}=1.004\right)$. The BSF is calculated at $662 \mathrm{keV}$ for ICRU, 1.10; and the PMMA phantom, 1.10, whereas for the PMMA phantom, it is $1.05 \pm 0.04$; and for the ISO water phantom, $1.03 \pm 0.04$ (Schauer et al., 2000) measured by an ionization chamber detector. The analysis shows that calibration of the TLD, Dosicard and Saphydose in freein-air exposure underestimates by $7.3-12 \%$.

We also found that the $B S F_{\text {PMMA }} \approx B S F_{\text {water }}$ for all the dosimeters, which shows that the density $\left(\mathrm{PMMA}=1.19 \mathrm{~g} \cdot \mathrm{cm}^{-3}\right.$ and water $\left.=1 \mathrm{~g} \cdot \mathrm{cm}^{-3}\right)$ differences of the material do not significantly affect the backscattering of photons, as demonstrated by MCNP 5a (X-5, 2003). The BSF relative to ICRU tissue for PMMA and water slab phantoms for the TLD is +1.4 to $+1.8 \%$; Dosicard, +1.1 to 
$+1.5 \%$; DRD, -9.1 to $-10 \%$ and Saphydose, -2.5 to $-3.7 \%$. The lower value of the $\mathrm{BSF}$ of the Saphydose may be set electronically to represent $H p(10)$ with less underestimation compared with the DRD. These BSFs of various dosimeters would be helpful for deriving the mechanism for equivalent dose measurement by measuring the ion beam or current by photon interaction.

\subsection{Impact of the BSF on $H p(10)$ measurement}

Equation (2) shows that measurement of $H p(10)$ is dependent on the response of the dosimeter to backscattered photons due to the mass attenuation coefficient of the phantom. Hence, for identical exposure conditions, the responses of the dosimeter depend upon multiple backscattered photons. The DRD is insensitive to backscattered photons by the phantoms; therefore, in-air exposure and on-body exposure are the same. If the exposure measured by the DRD is compared with the TLD or Dosicard calibrated in terms of $H p(10)$, a difference of $10-12 \%$ is expected in the reading, whereas a difference of around $3.4-5 \%$ is expected in the case of the Saphydose, which is less than half compared with the DRD. Even though both the Saphydose and Dosicard are calibrated in terms of $H p(10)$, the measurement may vary by around $5 \%$ due to differences in the response to the backscattered photons. Such variations would introduce systematic errors if not taken into consideration properly in institutions where multiple types of dosimeters are being used. The Saphydose response and dose evaluation results are better compared with the DRD, as it presents values of radiation exposure closer to the true dose. Since inherent accuracy of $\pm 10 \%$ of the exposure is a constraint of the dosimeter, underestimation of $H_{p}(10)$ is a combination of in-built and backscattering contribution. The uncertainty in $H p(10)$ is expected to vary in the range $-10.95 \pm 10 \%$, i.e. $-0.95 \%$ to $-20.95 \%$ compared with the true values of the exposure. This study reveals that the collective dose is expected to be underestimated by on average $20 \%$ by the DRD compared with the TLD.

\section{Conclusion}

This study highlights that the varying response of dosimeters due to backscattered photons indicates varying degrees of requirement of phantoms for measurement, and quality assurance of the dosimeters. It is evident that the response of the DRD to $H p(10)$ is nearly independent of the body or phantom, whereas the TLD, Dosicard and Saphydose require body-equivalent phantoms for calibration in terms of $H p(10)$ measurement. The BSFs of the dosimeters under study are comparable with established standard factors. The study also reveals that every institute/facility that uses different types of dosimeters for dose evaluation should establish the BSF of individual dosimeters for readily available correction factors 
for the calibration process to minimize the errors in the dose estimation. Our study resolves the ambiguity in individual dose measurement by various types of personal dosimeters by quantifying uncertainties due to backscattering factors.

Acknowledgement. The author (VPS) would like to thank Kaiga Atomic Power Station management for providing active personal dosimeters.

\section{REFERENCES}

Akar T.U., Ozmutlu E.N., Gurler O., Yalcin S. (2012) Monte Carlo analysis for multiple back scattering of gamma rays, J. Radioanal. Nucl. Energy, 295 (2), 901-905.

ANSI (1993) American National Standard Institute, American National Standard for Dosimetry, Personnel Dosimetry Performance, Criteria for Testing, ANSI N13.11.

Bartlett D.T., Dimbylow P.J., Francis T.M. (1980) Calculated Backscatter from Phantoms for Photon Dosemeter Calibration, Radiat. Prot. Dosim. 32 (2), 123-125.

Chan H.P., Doi K. (1960) Monte Carlo simulation studies of backscatter factors in mammography, Radiat. Res. 12 (1), 20-37.

Gualdrini G. (1993) Field parameters and Operational Quantities for ICRU sphere and reference photon beam, ENEA Report ISSN/1120-555.

Gualdrini G., Monteventi F., Morelli B. (1999) Determining the Photon Air Kerma Backscatter Factor Profiles for the ISO and ICRU Recommended Slab Phantoms: Comparison between LiF Measurements and Monte Carlo Calculations, Radiat. Prot. Dosim. 85 (1-4), 71-74.

Grosswendt B. (1990) Dependence of the photon backscatter factor for water on source-to-phantom distance and irradiation field size, Phys. Med. Biol. 35, 1233.

ICRU (1980) International Commission on Radiological Units and Measurements, Radiation Quantities and Units, Oxford, ICRU Report 33.

ICRU (1985) International Commission on Radiological Units and Measurements, Determination of Dose Equivalent Resulting from External Radiation Sources. Oxford, ICRU Report 39.

Kim C.H., Reece W.D., Poston J.W. (1999) Development of a Two-dosimeter Algorithm for Better Estimation of Effective Dose Equivalent and Effective Dose, Radiat. Prot. Dosim. 81 (2), 101-112.

Kramer R., Drexler G., Petoussi H.N., Zankl M., Regulla D., Panzer W. (2001) Backscatter factors for mammography calculated with Monte Carlo methods, Phys. Med. Biol. 46 (3), 771-781.

Lakshmana A.R., Kher R.K., Supe S.J. (1991) Estimation of Effective Dose Equivalent Using Individual Dosimeters, Radiat. Prot. Dosim. 35 (4), 247-252.

Mizukami K., Matsumoto T., Hydo T. (1967) Backscattering of gamma rays from polyethylene, alumium and lead slabs, J. Nucl. Sci. Tech. 4, 607-613.

Sabharwal A.D., Sandhu B.S., Singh B. (2009a) Investigations of energy dependence of saturation thickness of multiply backscattered gamma photons in carbon, Asian J. Chem. 21, 237-241.

Sabharwal A.D., Singh M., Singh B., Sandhu B.S. (2009b) Experimental evaluation of multiple Compton backscattering of gamma rays in copper, Indian J. Phys. 83, 1141-1146.

Schauer D.A., Cassata J.R., King J.J. (2000) A Comparison of Measured and Calculated Photon Backscatter from Dosemeter Calibration Phantoms, Radiat. Prot. Dosim. 88 (4), 319-321.

Scott O.S., Thomas F.G. (2008) Variations in backscatter observed in PMMA whole-body dosimetry slab phantoms, Radiat. Prot. Dosim. 128 (3), 375-381.

Selbach H.J., Grosswendt B., Hohfeld K., Kramer H.M. (1985) Experimental and Computational determination of the dose Equivalent Distribution in ICRU sphere, Radiat. Prot. Dosim. 12: 129-133. 
BACKSCATTERING FACTOR FOR SOME PERSONAL DOSIMETERS

Traub R.J., McDonal J.C., Murphy M.K. (1997) Detecrmination of Photon backscatter from several calibration phantoms, Radiat. Prot. Dosim. 74 (1-2), 13-20.

Vohra K.G., Bhatt R.C., Bhuwan C., Pradhan A.S., Lakshmanan A.R., Shastry S.S. (1980) A Personnel Dosimetry TLD Badge based on CaSO4: Dy Teflon TLD discs, Health Phys. 38, 193-197.

Will W. (1991) Measurement of conversion coefficient for calibration individual dosimeter with respect to the Operational dose quantities on the PMMA slab phantom, Radiat. Prot. Dosim. 37, 79-84.

X-5 (2003) Monte carlo Tem, MCNP-A general monte carlo n-particle transport code, version 5. University of California for U. S. Department of Energy. 